\title{
Towards Polymers with Molecular Auxeticity
}

\author{
Mahshid Alizadeha, Iris K. Tenniea, Ullrich Steiner ${ }^{b}$, and Andreas F. M. Kilbingerª
}

\begin{abstract}
For many decades, it has been challenging to synthesize auxetic materials at the molecular level. Auxetic materials exhibit counterintuitive behavior; they expand perpendicularly to the direction in which they are stretched. An aromatic macrocycle containing a sequence of $N$-substituted and $N$-unsubstituted amides was designed to resemble the re-entrant structure found in macromolecular auxetic materials. Upon application of tensile force, bent cis amides change to linear trans amides. This was anticipated to trigger the expansion of the macrocycle perpendicular to the direction of the applied force. To investigate the proposed configurational change by atom force microscopy (AFM), we designed and prepared a cis-trans aramide motif incorporated into an end-functionalized polymer which ensured covalent attachment to the AFM tip. At large extensions, polymer chains were envisioned to unfold and induce cis-trans isomerization.
\end{abstract}

Keywords: Auxetic materials · Configurational change · Poisson's ratio · Polymers · Re-entrant structures

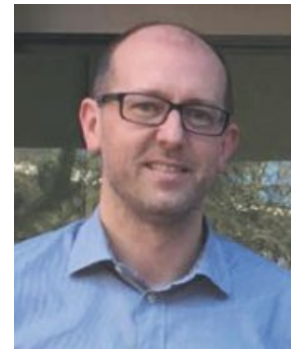

Andreas Kilbinger studied chemistry at the Johannes Gutenberg-Universität in Mainz and the Free University of Berlin in Germany (1991-1996) before working on his $\mathrm{PhD}$ (1996-1999) in polymer chemistry at the University of Durham in the United Kingdom (Prof. W. J. Feast). With a Feodor Lynen Fellowship he joined the group of Prof. R. H. Grubbs as a postdoctoral scholar at the California Institute of Technology (2000-2002) and then returned to the Johannes Gutenberg-Universität in Mainz for his habilitation (group of Prof. H. Frey) in organic chemistry (2007). In 2010 he joined the chemistry department at the University of Fribourg in Switzerland as a professor for polymer chemistry.

\section{Introduction}

Generally, when a material such as rubber is stretched or compressed at two opposite ends, it contracts or expands in the transverse direction to the applied force. This deformation can be quantified by Poisson's ratio $(v)$ which is defined as a negative ratio of perpendicular contraction strain to longitudinal expansion strain. The overwhelming majority of materials thus exhibit a positive Poisson's ratio. Some unusual materials, on the other hand, expand in at least one direction when stretched and contract when compressed. These materials display a negative Poisson's ratio and are denoted as auxetic materials. ${ }^{[1-4]}$ In nature, auxetic behavior can be found in cat skin, ${ }^{[5]}$ cow teat skin, ${ }^{[6]}$ iron pyrite ${ }^{[7]}$ and $\alpha$-cristobalite tetrahedral networks ${ }^{[8]}$ among others. Due to higher indentation resistance and energy absorption compared to the conventional materials, man-made macroscopic auxetic materials have been applied in surgical implants, ${ }^{[9]}$ smart bandages, ${ }^{[10]}$ artificial blood vessels, ${ }^{[11]}$ bulletproof vests, ${ }^{[12]}$ piezoelectric sensors, ${ }^{[13]}$ smart filters ${ }^{[14]}$ and sportswear. ${ }^{[15]}$

${ }^{\star}$ Correspondence: Prof. A. F. M. Kilbingera

E-mail: Andreas.Kilbinger@unifr.ch

aChemistry Department, University of Fribourg, Chemin du Musée 9 $\mathrm{CH}-1700$ Fribourg

${ }^{\text {b} A d o l p h e ~ M e r k l e ~ I n s t i t u t e, ~ C h e m i n ~ d e s ~ V e r d i e r s, ~} \mathrm{CH}-1700$ Fribourg
In $d$-dimensional isotropic elastic materials, Poisson's ratio is related by

$$
v=\frac{d K-2 G}{d(d-1) K+2 G}
$$

to the shear $(G)$ and bulk $(K)$ moduli, which are the measures of the rigidity and compressibility, respectively. ${ }^{[16]}$ Due to thermodynamic stability constraints, $K$ and $G$ must be positive in isotropic materials and so their Poisson's ratio must lie in the interval between -1 and $(d-1)^{-1}$. Since the properties of anisotropic materials are directionally dependent, the range of the Poisson's ratios in anisotropic materials is not restricted. ${ }^{[17]}$ The sign and magnitude of Poisson's ratio as well as its dependence on the strain are governed by the geometry and deformation mechanism of the material architecture on a variety of length scales. ${ }^{[18]}$

For example, a honeycomb network contracts laterally when stretched axially (Fig. 1A), which results in a positive Poisson's ratio. In 1987, Lakes suggested and designed thermoplastic foams that exploit re-entrant or inwardly directed honeycomb microstructure (Fig. 1B). ${ }^{[19]}$ Upon stretching in longitudinal direction, the hinging ribs of the hexagonal cells open, and the cells undergo elongation not only in longitudinal but also in transverse direction to the applied load. The degree of auxeticity in re-entrant honeycombs can be regulated by adjusting the negative angle of the re-entrant and the ratio of the side lengths of the cell. ${ }^{[20]}$

Other re-entrant honeycomb structures include arrowheads (Fig. 2A), whereby compression in the longitudinal direction results in a collapse of the triangles in the transverse direction. ${ }^{[21]}$ The Poisson's ratio of rotating (semi-)rigid squares, rectangles (Fig. 2B), triangles, rhombi and parallelograms connected at the vertices also strongly depends on the angle of the hinges. ${ }^{[22]} \mathrm{On}$ the other hand, the Poisson's ratio of chiral structures (Fig. 2C) does not rely on re-entrant angles since a cell unit consists of nodes connected by ligaments in the form of either conventional or re-entrant hexagonal honeycombs which rotate and flex the ligaments in response to compression. This deformation mechanism enables them to maintain their auxetic behavior over a larger range of strain. ${ }^{[23]}$ Void space of a cell unit is, however, not a prerequisite for auxeticity. An example of voidless materials are 
Conventional materia
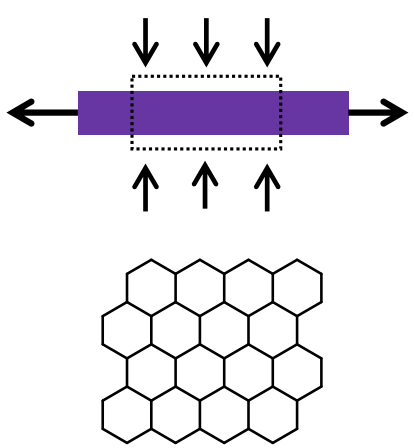

A
Auxetic material
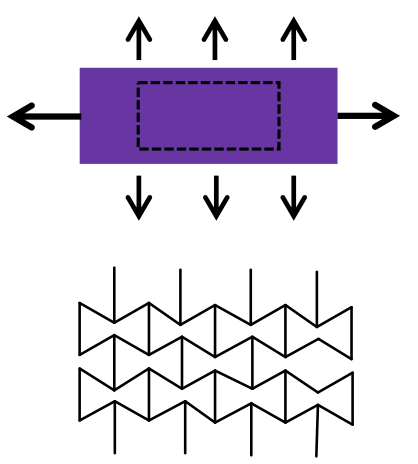

B
Fig 1. Comparison of conventional and auxetic materials exemplified by hexagonal honeycomb $(A)$ and re-entrant honeycomb $(B)$ networks, respectively.

hierarchical laminates (Fig. 2D) composed of segments with two different Young's moduli, of which one must be less than about $5 \%$ of the modulus of the stiff component in order to contribute to a negative Poisson's ratio. ${ }^{[24]}$ Similarly, non-porous materials close to a phase transition such as the $\alpha-\beta$ transition in polycrystalline quartz exhibit a weak negative Poisson's ratio.[17]

Many attempts were made to reproduce macromolecular auxetic materials at the molecular scale. In 1992, Evans et al. designed and modelled 2-dimensional molecular networks based on a reentrant honeycomb structure with joints composed of benzene and straight lines comprised of acetylene links. ${ }^{[20]}$ Similarly, 2D selfassembled polymers consisting of hard double-arrow-like pentaphenyl segments and various soft spacers were predicted by molecular simulation to possess negative Poisson's ratios. ${ }^{[25]}$ Despite numerous theoretical proposals, such materials have never been synthesized. ${ }^{[26]}$ Honeycomb-like sheets with cavities of about 10 $\AA$ in diameter, on the other hand, were prepared by Gardner and colleagues. However, this self-assembled hinged network based on $\mathrm{Ag} \ldots \mathrm{N}$ coordination bonds between alternating 1,3,5-tricyanobenzene and silver triflate units has also only been predicted but never proven to be truly auxetic. ${ }^{[27]}$

Another route to the molecular auxetic subunits was envisioned by Griffin and others, who integrated either terphenyl or pentaphenyl moieties into the backbone of liquid crystalline aromatic polyesters. Oligophenyl rods connected laterally by flex- ible spacers were supposed to rotate to the transverse direction, push neighboring chains apart and cause volume expansion upon stretching. While the rigid rod rotation driven by tensile force was indeed confirmed at the molecular level by X-ray diffraction, only positive Poisson's ratios were observed at the macromolecular level possibly due to the slippage of polymer backbone layers under tensile load.[28]

\section{Potentially Auxetic Polymers Based on Aramides}

As the geometry of the repeat unit plays a crucial role for the manifestation of auxetic behavior in materials, shape-persistent rigid aromatic amides known as aramides were chosen to represent a re-entrant structure unit (Fig. 3A). Since $N$-substituted amides naturally adopt a cis configuration, a sequence of $N$-substituted and $N$-unsubstituted amides forces the macrocycle to assume the re-entrant structure. The bent geometry of cis amides was envisioned to transform into linear trans amide configuration upon application of tensile force at the lateral hydroxyl groups (Fig. 3A).

Based on our group's expertise in the field of the sequencecontrolled assembly of oligo( $p$-benzamide)s, ${ }^{[29]}$ we prepared oligomers/polymers containing cis-aramide motifs to investigate the proposed configurational change by fluorescence spectroscopy and atomic force microscopy (AFM).

The cis-trans amide isomerization in aromatic amide oligomers was confirmed by fluorescence spectroscopy. For this purpose, the end groups of sequential $N$-substituted and $N$-protective unsubstituted amides, which form V-shaped segments, were labelled by pyrene. In the cis-configuration, the chromophores were in close proximity to each other, whereas in the trans-configuration the distance between the chromophores increased (Fig. 3C). These changes could be followed by the disappearance of the pyrene excimer signal in the fluorescence spectrum. By taking advantage of the $N$-protective amide as an acid labile group, the protective group could be cleaved off in an acid medium and cis-trans isomerization occurred. This resulted in an increased distance between the pyrene chromophores with a concurrent reduction in pyrene excimer fluorescence intensity.

The $N$-unsubstituted and $N$-alkyl-substituted aramides, which can undergo cis-trans isomerization (Fig. 3B), were also incorporated into a methyl acrylate polymer. To ensure the covalent attachment of the polymer to the surface and AFM tip, we appended a second block copolymer carrying amine end groups. Upon application of tensile force, polymer chains were expected to uncoil, while a stronger force was anticipated to induce the
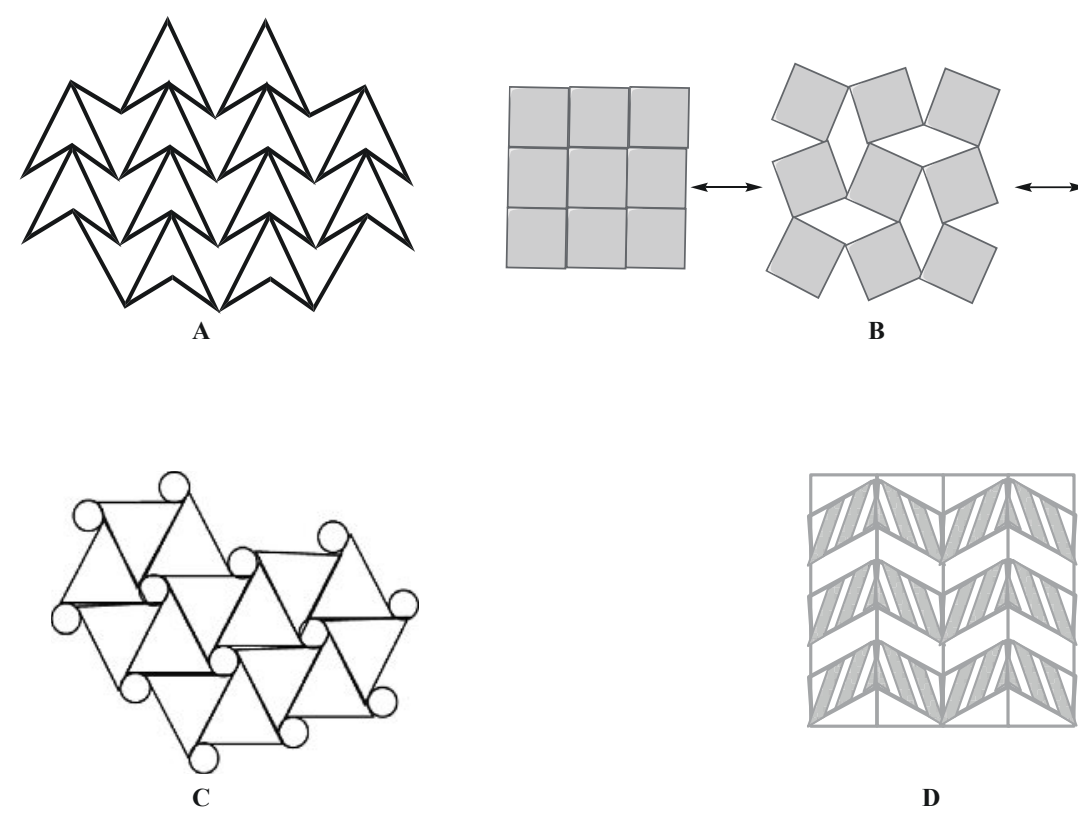

Fig 2. Double arrowhead reentrant structure (A). Rotating squares (B). Chiral honeycomb structure (C). Auxetic composites based on hierarchical laminate structure (D). 


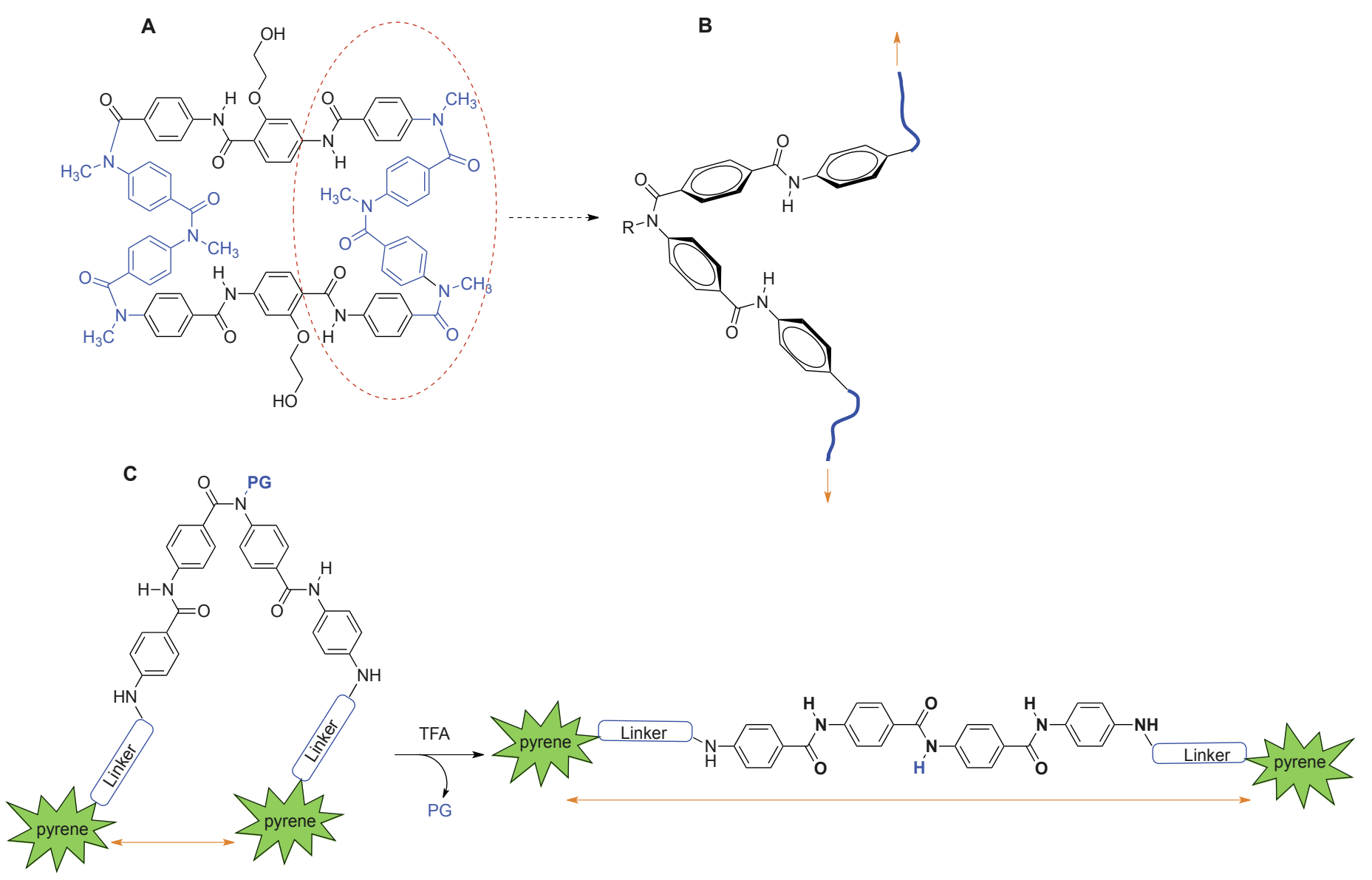

Fig. 3. A) Chemical structure of a re-entrant oligoaramide macrocyclic unit. Blue and black colors indicate cis- and trans-segments of the aramides, respectively. B) Model polymer containing aramide segments undergo a reversible cis-trans isomerization upon application of force. C) Pyrenelabelled aramide oligomers undergo an irreversible cis-trans isomerization, which is triggered by cleavage of the protective group in the presence of trifluoroacetic acid (TFA).

reversible cis-trans isomerization thus causing a distinct spike in the force-distance curve. The number of $N$-alkyl substituted amide segments was anticipated to influence the magnitude of the applied force required to isomerize cis to trans amide. Also, the placement of cis segments between rigid rod-like linear aramides should substantially reduce the force magnitude necessary to isomerize cis to trans amide. In order to show the difference in the applied force needed for the cis to trans isomerization, we synthesized a trimer as a shorter and a tetramer as a longer aramide. AFM measurements of both polymer models containing trimer and tetramer rigid aramides are currently under investigation by Prof. Michal Borkovec's group at the University of Geneva.

\section{On the Conceptual Difficulty in Manufacturing Molecular Auxetic Materials}

Eqn. (1) illustrates the obstacles in the manufacture of molecular organic auxetic materials. In most polymers, $G<<K$, which implies $v \approx 1 / 2$. In order to lower $v, G / K$ has to be raised. For example, $G=1.5 \mathrm{~K}$ for $\mathrm{v}=0$, and therefore $G$ must significantly exceed $K$ to achieve negative $v$ values. In all known polymers, however, $G \leq K$, which explains the experimentally observed Poisson's ratios between $1 / 3$ and $1 / 2$. While polymer melts almost invariably have $v \approx 1 / 2$, glassy polymers tend to have Poisson's ratios below 1/2 (but above 1/3), because of their high shear moduli.

As already mentioned, Eqn. (1) does not apply to anisotropic systems. In terms of molecular approaches, this however not only requires an anisotropic molecular structure but an anisotropic assembly of these molecules on mesoscopic or macroscopic length scales. To illustrate this, it is instructive to start with the mechanical properties of isotropic polyisoprene, as a reference system for a polymer melt above its glass transition temperature, with an elastic modulus of $Y \sim 100 \mathrm{kPa}$ and a bulk modulus of $K \sim 1 \mathrm{GPa}$. In a hypothetical rubber-like material with $v=0$ (the cross-over case for negative $v$-values), the relative volume change $\Delta V / V$ directly mirrors the engineering strain $\Delta L / L$.

If volume is not conserved upon deformation (i.e. $v<0.5$ ), this implies that work has to be done against the cohesion of the molecules that make up the material, that is, energy has to be supplied to overcome the cohesive energy density defined as the ratio of cohesion energy $E_{\mathrm{c}}$ to the molecular volume $V_{\text {mol }}$. It is evident that $E_{c} / V_{\text {mol }}$ must be related to the bulk modulus $K$, which defines the resistance of the material against hydrostatic compression. For metals, $K=C E_{\mathrm{c}} / V_{\text {mol }}$, where $C$ is a constant that takes into account the packing density of the lattice atoms. ${ }^{[30]}$ By extrapolation, $C$ must be on the order of unity in low density disordered solids. Taking the value of polyisoprene, $K \sim 1 \mathrm{GPa}$, and a polymer volume of $1 \mathrm{~nm}^{3}$, this results in a value of $E_{\mathrm{c}}$ of $\sim 10^{-18} \mathrm{~J}$, which is on the order of several hundred $\mathrm{k} T$. The mechanical energy that is input by stretching and compression, is quantified by Young's modulus (which is also an energy density). Again, calculated for a molecular volume of $1 \mathrm{~nm}^{3}$, this results in an energy input on the molecular level of $10^{-22} \mathrm{~J}$, which is less than $\mathrm{k} T$. This back-ofthe-envelope calculation implies that additional work is required on the molecular level to substantially increase the molecular volume, which is necessary to lower the Poisson's ratio in isotropic solids below $v=1 / 2$. This energy is on the order of bond energies in organic materials of 150-400 kT. Changes in molecular conformation that are often invoked in the concepts of auxetic materials are associated with energy changes that are much below these bond energies, and therefore 1-2 orders of magnitude below the limit imposed by the high incompressibility in these materials. 
To understand the differences between hard crystalline materials (for some of which auxetic behavior is observed) and organic materials, it is instructive to revisit the molecular origin of the elastic (or shear) modulus and the bulk modulus in these two material classes. In inorganic crystals, both arise from a displacement of atoms in the lattice (i.e. the stretching of atomic bonds). In rubbers however, the elastic modulus arises from conformational entropy changes of deforming polymer chains from their lowest free energy coil conformation, while the bulk modulus still requires work against the intermolecular potentials (which are often of the van-der-Waals type). Since the former is much lower than the latter, this difference in mechanisms explains the large difference in elastic and bulk moduli in these systems, and equivalently, the difficulty in attaining Poisson's ratios much below $1 / 2$.

These simple considerations unify established experimental and conceptual observations, including (1) the incompressibility of polymer melts and rubbers, (2) the observation of Poisson's ratios close to $1 / 2$ in polymer rubbers, and (3) the observed requirement for design structures on macroscopic or mesoscopic length scales, the deformation of which results in low $v$-values. Returning to Eqn. (1), this argument also provides a molecular reasoning for the $K<G$ requirement to obtain negative Poisson's ratios.

We return to the question whether molecular auxetic materials are attainable. In the context of the above considered liquid incompressibility, it seems that strategies building on asymmetric molecular conformations alone are difficult. It might, however, be possible to design materials that exhibit collective transitions. In fact, phase transitions are known to lead to marked volume changes. Mechanically triggered phase transitions, such as the reversible crystallization or macroscopic packing of molecular units may yield the desired effects. In these processes, simple molecular reorganizations by themselves are however probably insufficient, but must be supported by collective free energy changes that are sufficiently powerful to drive the required volume changes. Ideally, these phase transitions should be reversible upon actuation. Other strategies yielding substantial volume changes are mesostructural reorganizations, such as the alignment of crystalline domains within semicrystalline materials, but it is unlikely that these processes are reversible.

Beyond this, two strategies emerge. One ensures the incompressibility of the materials by reversible liquid ingress, such as in a gel. The other involves the down-scaling of the plethora of structure conformation strategies in porous or composite systems to mesoscopic length scales below $1 \mu \mathrm{m}$. In this regime, self-organized mesoscopic or self-assembled molecular materials may provide interesting opportunities.

\section{Conclusion and Outlook}

Any isotropic auxetic material lowers its apparent density upon being stretched and increases its apparent density upon being compressed. Macroscopic auxetic materials are typically based on periodic patterns that change their macroscopic porosity upon deformation. In most cases, fluid (gas or liquid) exchange between the pores and the surrounding environment is crucial for auxeticity to be observable.

In any hypothetical molecular isotropic auxetic (bulk) material it is the real density of the material that must decrease or increase upon stretching or compression. In the absence of mass flow in or out of the material this can only be achieved by increasing or decreasing the volume of voids (i.e. vacuum) inside the material.

To mimic the large deformations observable for macroscopic auxetic patterns on the molecular level, it is tempting to exploit polymeric elastomers which can be stretched and compressed reversibly. Such rubber-like behavior is typically observed above the glass transition temperature of its constituting polymers, al- lowing for large segmental movements within the elastomeric material. Large conformational rearrangements, however, might be counterproductive when the creation and the structural support of voids is concerned, and for typical bulk moduli of these materials, large volume changes required unrealistic molecular free energy changes. On the other hand, the dynamic increase/ decrease of void volume upon application of external force might be feasible for rigid and highly ordered materials. Porous crystalline materials such as metal organic (MOF) or covalent organic frameworks (COF) could be suitable candidates even though their extent of elastic deformation will be greatly reduced compared to rubber-like elastomers.

While for elastomeric polymer networks the bulk state appears unlikely to support isotropic auxetic behavior, the situation might be reversed for the solvent swollen state (i.e. in a gel). There, the solvent can easily be exchanged between the surrounding environment and the pores of the network. If a material can be designed that changes the pore size or polymer-solvent interaction upon application of an external mechanical force, solvent influx or outflux might be triggered resulting in expansion or contraction of the polymer gel. In this manner, molecular isotropic auxetic behavior might be achievable for solvent swollen polymer networks.

\section{Acknowledgements}

The authors thank the Swiss National Science Foundation and the National Center for Competence in Research (NCCR) 'Bio-Inspired Materials' for financial support.

Received. November 16, 2018

[1] Y. Li, Phys. Stat. Soil. 1976, 38, 171.

[2] R. H. Baughman, Nature 2003, 425, 667.

[3] K. E. Evans, K. L. Alderson, Eng. Sci. and Edu. J. 2000, 9, 148

[4] Y. Prawoto, Comput. Mater. Sci. 2012, 58, 140.

[5] D. R. Veronda, R. A. Westman, J. Biomechanics. 1970, 3, 111

[6] C. Lees, J. F. V. Vincent, J. E. Hillerton, Bio-Med. Mater. Eng. 1991, 1, 19.

[7] A. E. H. Love, 'A Treatise on Mathematical Theory of Elasticity', 4th Ed., Dover, New York, 1944.

[8] A. Yeganeh-Haeri, D. J. Weidner, J. B. Parise, Science 1992, 257, 650.

[9] C. Koerner, P. Heinl, R. F. Singer, U.S. Pat. Appl.No.: 12/998,610, 2008.

[10] A. Alderson, J. Rasburn, S. Ameer-Beg, P. G. Mullarkey, W. Perrie, K. E. Evans, Ind. Eng. Chem. Res. 2000, 654.

[11] J. Chlupac, E. Filova, L. Bacakova, Physiol. Res. 2009, 58,119.

[12] Z. Y. Wang, H. Hu, Phys. Status. Solidi. B 2013, 251, 281.

[13] W. A. Smith, V. Vienna, Patent US5334903, USA, 1994.

[14] A. Alderson, J. Rasburn, S. Ameer-Beg, P. Mullarkey, W. Perrie, E. Evans, Ind. Eng. Chem. Res. 2000, 39, 654.

[15] T. M. Cross, K. W. Hoffer, D. Jones, P. B. Kirschner, E. Langvin. Sherwood, J. C. Meschter, US 9.402.439 B2, 2016.

[16] V. F. Hagh, M. F. Thorpe, Phys. Rev. B 2018, 98, 100101.

[17] R. S. Lakes, Annu. Rev. Mater. Res. 2017, 47, 63.

[18] K. E. Evans, A. Alderson, Adv. Mater. 2000, 12, 617.

[19] R. S. Lakes, Science 1987, 235, 1038.

[20] a) K. E. Evans, M. A. Nkansah, I. J. Hutchinson, S. C. Rogers, Nature 1991, 353, 124; b) B. D. Caddock, K. E. Evans, J. Phys. D: Appl. Phys. 1989, 22, 1877.

[21] U. D. Larsen, O. Sigmund, S. Bouwstra, J. Microelectromech. Syst. 1997, 6, 99.

[22] J. N. Grima, K. E. Evans J. Mater. Sci. Lett. 2000, 19, 1563.

[23] H. M. Kolken, A. A. Zadpoor, RSC Advances 2017, 7, 5111

[24] S. V. Shilko, E. M. Petrokovets, Y. M. Pleskachevsky, Phys. Stat. Sol. 2008, $245,591$.

[25] G.Wei, Phys. Stat. Soil. B 2005, 242, 742.

[26] F. Porzio, É. Cuierrier, C. Wespiser, S. Tesson, R. S. Underhill, A. Soldera, Mol.Simul. 2017, 43, 169.

[27] G. B. Gardner, D. Venkataraman, J. S. Moore, S. Lee, Nature 1995, 374, 792.

[28] a) C. B. He, P. W. Liu , A. C. Griffin, Macromolecules 1998, 31, 3145; b) C. B. He, P. W. Liu , P. I. McMullan, A.C. Griffin, Phys. Stat. Sol. 2005, 242, 576.

[29] a) H. Seyler, A. F. M. Kilbinger, Macromolecules 2010, 43, 5659; b) H. Seyler, A. F. M Kilbinger, Macromolecules 2009, 42, 9141.

[30] S. Wacke, T. Górecki, Cz. Górecki, K. Książek, J. Phys.: Conf. Ser. 2011, 289,012020 